\title{
Geschichte des Public Law als Staatsphilosophie
}

\author{
Miloš Vec" \\ Rezension zu Martin Loughlin, Foundations of Public Law, Oxford University Press, Oxford \\ 2010, 515 S., gebunden ca. 120 \$, ISBN 978-0-19-925685-3
}

Martin Loughlins Monografie ist ein eigenwilliges Buch, das zwischen Geschichte, Theorie und Philosophie des „public law“ oszilliert. Der Verfasser knüpft dabei vielfach an Vorarbeiten an, besonders an seine 2003 erschienene Studie „The Idea of Public Law“. Der Titel „Foundations of public law” ist erneut schlicht, aber insofern passend gewählt, als er die genaue Stoßrichtung zwischen theoretischen und historischen Grundlagen bewusst offen lässt. Die folgende Kritik konzentriert sich auf die rechtsgeschichtlichen Aspekte des Werks und liest es insofern aus Sicht des Autors möglicherweise einseitig; zugleich ist Loughlins Buch aber auch keine Zurückweisung einer solchen rechtsgeschichtlichen Lektüre zu entnehmen. Das Buch ist teilweise in Deutschland entstanden, Loughlin verbrachte das akademische Jahr 2007/2008 am Wissenschaftskolleg zu Berlin und war dort Mitglied der Schwerpunktgruppe „Verfassung jenseits des Nationalstaates“ zusammen mit Petra Dobner, Dieter Grimm, Bogdan Iancu, Fritz Scharpf, Alexander Somek, Gunther Teubner und Rainer Wahl. Loughlin ist Professor of Public Law an der London School of Economics and Political Science. Das ist für das Verständnis seines Anliegens wichtig, da England keinen Staat im kontinentalen Sinne ausgebildet hat und es dort auch keine lebendige public law-Tradition gibt. Loughlin interessiert sich für die Gründe der Verdrängung und versucht, das verloren gegangene juristische Feld wissenschaftlich zu revitalisieren.

Das Buch setzt schwungvoll an und erläutert dem Leser den Gegenstand seines Interesses. Das heutige „public law“ sei analog zu dem, was mittelalterliche Juristen „fundamental law“ genannt hätten (1). In die modernen Sprachen übersetzt, lasse sich des Verfassers Interesse am „public law“ als jenes an den „Principes du droit politique“ bzw. „Grundlagen des (allgemeinen) Staatsrechts“ paraphrasieren (3). Das klingt nicht von ungefähr nach Standardwerken aus der Geschichte des öffentlichen Rechts; tatsächlich werden Rousseau, Gerber und andere Klassiker vielfach herangezogen und ausgelegt. Später lautet die Gleichsetzung „public law“ = „droit politique“ = „Staatsrecht“ (231). Die historische Spanne reicht dabei in etwa von Bodin bis in das späte 19. Jahrhundert, wo die Quellenstudien bei Paul Laband, Otto Mayer und Albert Venn Dicey ausklingen; aber auch Carl Schmitt spielt noch eine große Rolle. Die Lektüre dieser „Political Jurisprudence“ (9, 157 ff.) erfolgt aber

* PD Dr. Miloš Vec ist am Max-Planck-Institut für europäische Rechtsgeschichte in Frankfurt am Main tätig und wird ab Oktober 2012 die Professur für europäische Rechts- und Verfassungsgeschichte an der Universität Wien übernehmen. 
offenbar nicht aus einem rein wissenschaftsgeschichtlichen Interesse, Loughlin verwendet seine Quellen vielmehr in einer universalisierenden Weise, er interessiert sich für „the nature of public law" (208) und dessen „Grammatik“ (178 ff.). Eine solche Sprechweise von der "distinctive nature of public law" (6), „the nature of political right" (11) und „the distinctive nature of political power" (11) setzen voraus, dass es ein solches „Wesen“ des „public law“, des „political right“ oder der „political power" gibt, was man als Prämisse nicht unbedingt teilen muss. Die historischen Quellen werden mithin als Bausteine für eine anhaltende Regulierungsherausforderung jeglicher Staatlichkeit geprüft; entsprechend steht Verwendbarkeit im Vordergrund, nicht deren Zeitgebundenheit. Anders gesagt: Philosophie und Theorie der „politischen Rechtsgelehrsamkeit“ überlagern deren Historizität.

Loughlin erzählt verschiedene miteinander verknüpfte Entwicklungs- und Fortschrittsgeschichten des Diskurses. Im Zentrum dieses Diskurses steht ein Objekt, das leider seltsam unscharf bleibt. Es ist der „Staat“. Vom Staat und der Staatlichkeit ist viel die Rede, dabei bleibt der Abstraktionsgrad aber recht hoch. Loughlins Interesse an den Regulierungsherausforderungen spiegelt sich in der Sprache der theoretisierenden Quellen, die Ideen formulieren, Grundsätze aufstellen und Prinzipien miteinander in Einklang bringen möchte. Sehr in die regulatorischen Details geht Loughlin dabei nicht, was auch daran liegen mag, dass die Zeitgebundenheit der Entwürfe einer solchen überzeitlichen Lektüre im Wege steht. Was die Kantische Rechts-StaatsSchule für richtig hielt, was Robert von Mohl postulierte (318 f.), ist vor den Folien historisch gewordener Rechts- und Verfassungsordnungen zu sehen. Heutige Rechtsstaatsdiskussionen stehen teils vor ähnlichen, teils vor deutlich anderen Herausforderungen.

So ist das Buch auf eine merkwürdige Weise staatsfixiert im Sinne und in der Tradition des 19. Jahrhunderts. Loughlin interessiert sich für die Innenseite der Herrschaft, externe Beziehungen des Nationalstaats oder Verflechtungen zwischen Innen und Außen kommen nicht oder nur marginal vor. Völkerrecht und Diplomatie sind jenseits des politisch-juristischen Horizonts. Auch die politischen Entitäten unterhalb der Ebene des Nationalstaats werden vernachlässigt: Kommunen, Länder und /oder Bundesstaaten tauchen auf der juristischen Landkarte nicht auf. Loughlins Begriff des „public law“ exkludiert demnach nicht nur international public law, sondern über weite Strecken auch das Verwaltungsrecht oder kommunale Satzungen. Weil Loughlins Interesse nicht genuin historisch ist, kommt auch die Fülle der vormodernen Policey nicht in den Blick, weder im Sinne der Gesetzgebung und des Policeyrechts noch in der zeitgenössischen politischen Theorie, die sich dem ausführlich widmete. Der teleologische Blick übergeht jene vormodernen Materien, die auf dem Weg zur modernen Staatlichkeit untergegangen sind oder abgespalten wurden. 
Die Darstellungsweise ist zunächst historisch, das heißt, Loughlin entwickelt in Part I zunächst „Medieval Origins“ (17ff.) des public law, wie es sich parallel zur Entstehung des modernen Staates ausgebildet hat. Er führt den Leser von einem kanonischen geprägten Recht und theologischen Grundlagen politischer Herrschaft zum säkular verstandenen Naturrecht und zum „Modern Constitutionalism“ (46ff.). Hier wie später bei den Ausführungen zu „The Birth of Public Law“ (50 ff.) werden inhaltliche Axiome und methodologische Grundlagen referiert, analysiert und kritisiert. Die Überschriften sind knapp und manchmal vielleicht etwas zu formal angelegt. Man könnte dem Leser auch programmatisch in der Gliederung sagen, worin „The Methodological Turn“ (51-55) oder „Bodin's Method“ (56-62) bestand. „Absolutism“ wiederum (62-69) ist jedenfalls unter Frühneuzeit-Historikern eine problematisch gewordene Kategorie, wovon der Leser aber nichts erfährt. Erst recht wirken aufeinanderfolgende Kapitelüberschriften mit Titeln wie „The Science of Political Right: I“ und „The Science of Political Right: II“ blass und inhaltlich ziellos. Dieser historische Aufbau wird aber nicht für das ganze Buch durchgehalten, sondern wiederholt sich en miniature in den Einzelabschnitten. Im Vordergrund stehen demnach einzelne Problemkomplexe des "public law“, innerhalb derer die politisch-juristische Theorie historisch aufgerollt wird. Entsprechend findet man die Großtheoretiker der Staatlichkeit und des Staatsrechts wie Bodin, Hobbes oder Locke über das ganze Buch verstreut. Leider ist das Register weder bei Personennamen noch bei Sachmaterien wirklich zuverlässig.

Die Analyse der Problemkomplexe erfolgt als ideengeschichtliche „Gipfelwanderung " unter den kanonisierten (west-)europäischen Theoretikern. Konkrete Institutionen, Verfassungsnormen kommen selten vor (222, 255, 282 ff., 346, 353 und öfter), einfaches Gesetzesrecht oder die Rechtsprechung gleichfalls nur peripher. Die Rückbindung an die Realgeschichte in allen ihren Facetten ist also schwach. Loughlin zieht stattdessen hauptsächlich in transparenter, aber auch erwartbarer Weise die bekannten Staatstheoretiker heran und führt sie zu fiktiven Dialogen zusammen. So geraten Gelehrte aus verschiedenen Jahrhunderten miteinander ins Gespräch zu jenen Sachkomplexen der politischen Philosophie, die Loughlin besonders interessieren. Es sind immer deren Hauptwerke, und Loughlin unternimmt Exegesen, die gleichermaßen Quellenstudien und Forschungsüberblicke umfassen. Er stützt sich vor allem auf englische, französische und US-amerikanische Meisterdenker. Der Sprachpluralismus, den seine erste Fußnote auf Seite 1 verheißt, wo sich passende Forschungsliteratur aus drei Ländern verdichtet findet, wird aber nicht durchgehalten. Zur Begriffsgeschichte der „Constitution“ (275 ff.) wäre ein Hinweis auf die Monografie Mohnhaupt/ Grimm wünschenswert gewesen. Auch die erfreuliche Erwähnung der Ersterscheinungsjahre bei den Quellen in Form von Zusätzen in eckigen Klammern „Leviathan [1651]“ oder „Heller, Staatslehre [1934]“ (234) wird nicht systematisch durchgeführt. 
Im Gegenteil, der Umgang mit Quellen und Forschungsliteratur erscheint bei zunehmender Lektüre nachlässig und problematisch. Zunächst sei das Sprachproblem notiert. Selbst die Zelebritäten unter den Franzosen wie Bodin und Montesquieu (454) werden nach englischen Ausgaben zitiert, deutsche Autoren sowieso: Pufendorf und Hegel, Max Weber, Ernst Fraenkel und viele andere mehr. Immerhin gibt es in seltenen Fällen in den Fußnoten die originalen Quellenzitate und parallele Übersetzungen (Gerber, 192; Jellinek, 193); ein Muster war mir hier nicht erkennbar. Hinzu kommt der systematische Zugriff auf bestimmte Gattungen von Büchern, die nicht immer adäquat scheinen. Denn gerade die Engländer und Amerikaner selbst werden fast ausschließlich nach populären Studienausgaben zitiert (Paine 159, Hobbes 172, 214, Hume 220). Zumal bei den deutschen Autoren wiederum erweisen sich die genauen Referenzen als fehlerhaft. Pufendorfs „Die Verfassung des Deutschen Reiches" von 1667 war - anders als es die Zitierweise (79f.) suggeriert - keine deutschsprachige Schrift. Bei den Autorennamen sind die Schreibweisen von Lübbe (50), Dreitzel (79), North (166) und anderen fehlerhaft. Bei anderen trifft es die Titel ihrer Publikationen oder die herangezogenen Zitate, so vielfach gerade bei den deutschsprachigen Autoren: Schnur (69), Bluntschli (185), Rotteck (190), Kelsen (211), Dahrendorf (219), Heller und Gerber (234), Welcker (318), Forsthoff (461), Jellinek (458), Schellenberg (313), etc. Als Leser wüsste man gerne, ob und welche Standards ein großer und renommierter Verlag wie Oxford University Press wissenschaftlichen Werken auferlegt, ob etwa Sprachenpluralismus selbst bei so gelehrten, historischen Studien, die sich an ein Fachpublikum wenden, als unerwünscht zurückgewiesen wird. Auch wären die Standards und ihre praktische Implementation im Lektorat ein Innehalten wert.

Loughlins Buch hat demnach als Wissenschaftsgeschichte eines bestimmten Ausschnitts des öffentlichen Rechts in der Entstehungsphase zwischen ca. 1600 und 1900 seine handwerklichen Fehler und systematische Schwächen im Aufbau; inhaltlich kann die Darstellung keine Originalität beanspruchen und will es auf diesem rechtshistorischen Feld vermutlich auch nicht. Zumal für den deutschen Leser liegt der Rückgriff eher auf die vorhandenen Standardwerke nahe. Die politische Philosophie wiederum, die Loughlin dem Leser in dieser historischen Gewandung nahebringen will, ist diffus, seine Beweisführung alles andere als klar artikuliert. Sie zu beurteilen fällt vermutlich eher in die Zuständigkeit anderer Rezensenten. Loughlins Denkstil scheint auf das Aufzeigen von Widersprüchlichkeiten hinauszulaufen, wobei er gerne zu indifferenten Schlüssen kommt: „dilemma“ $(13,429)$, „basic tension“ (197), „paradox“ (78, 83 ff., 94, 116 f., 151, 226, 232, 269, 311), „tension“ $(23,85,88,95,103,107,119,163,234,236,282,302,319,321,370,375,383$, 430 und öfters), „mutual tension“ (272) sind die Zentralbegriffe, mit denen er juristisch-politische Relationen charakterisiert, die Entwicklungspfade laufen entlang der Meistererzählungen von „historicization“ (50), „modernization“ (7, 9, 243, 259, 
376, 311, 379, 392, 434, 437 und öfter), „rationalization“ $(2,50,133,414,434$, 436 f., 463), „secularization“ (37 ff., 50, 185), „formalization“ (157, 265, 316, 319, 322, 429) und „positivization“ (2, 7, 10, 109, 151, 297, 311). Als Metakonflikt macht Loughlin jenen zwischen dem Wunsch nach individueller Freiheit/ Autonomie und dem Wunsch nach Teilhabe an Gemeinschaft aus (11). Dass der Diskurs über politische Rechte, der sich aus diesem Spannungsverhältnis ergibt, gleichermaßen „enabling and constraining“ sei (12), ist schließlich auch als Einsicht wenig überraschend, jedenfalls aus rechtshistorischer Sicht. Ob die Staatsphilosophie Loughlins Buch anders sieht, müsste diese sagen. So bietet „Foundations of public law” primär einen schwungvollen Überblick und einen leicht fasslichen Einstieg in ein enorm weites Feld. Die eigentliche Leistung und das Verdienst Loughlins sind die Übersetzung und der Import einer reichen kontinentaleuropäischen Staatstheorie in einen Common Law-Kontext, der mit diesen Autoren und Ideen immer noch zu wenig vertraut ist. 